\title{
Interleukin-2, Interferon-gamma Gene Polymorphisms in Recurrent Aphthous Stomatitis
}

\author{
Shamsolmoulouk Najafi ${ }^{1}$, Hila Yousefi ${ }^{2,3}$, Mahsa Mohammadzadeh ${ }^{1,4}$, \\ Alireza Zare Bidoki ${ }^{5}$, Elham Farhadi ${ }^{6}$, Nima Rezaei ${ }^{2,5,7}$ \\ ${ }^{1}$ Department of Oral Medicine, School of Dentistry, Tehran University \\ of Medical Sciences, Tehran, Iran; \\ ${ }^{2}$ Research Center for Immunodeficiencies, Children's Medical Center, Tehran \\ University of Medical Sciences, Tehran, Iran; \\ ${ }^{3}$ Department of Endodontics, Dental Branch, Islamic Azad University, Tehran, Iran; \\ ${ }^{4}$ Department of Orthodontics, Dental Branch, Islamic Azad University, Tehran, Iran; \\ ${ }^{5}$ Department of Immunology, School of Medicine, Tehran University of Medical \\ Sciences, Tehran, Iran; \\ ${ }^{6}$ Department of Hematology, School of Allied Medical Science, Iran University \\ of Medical Sciences, Tehran, Iran; \\ ${ }^{7}$ Network of Immunity in Infection, Malignancy and Autoimmunity (NIIMA), \\ Universal Scientific Education and Research Network (USERN), Sheffield, UK
}

Received April 7, 2017; Accepted August 28, 2017.

Key words: Recurrent aphthous stomatitis - Interleukin-2 - Interferon-gamma Single nucleotide polymorphisms

\begin{abstract}
Recurrent aphthous stomatitis (RAS) is the most common oral ulcerative inflammatory disease with unknown etiology. IL-2 and IFN- $\gamma$ are secreted by Th1 cells and the elevated levels of them have been reported in RAS. Single nucleotide polymorphisms (SNPs) of IL-2 and IFN- $\gamma$ genes could alter the cytokine production. The aim of this study was to investigate frequencies of IL-2 and IFN- $\gamma$ alleles and genotypes in a group of patients with minor-RAS (MiRAS). PCR-SSP method used to type genomic DNA of 64 Iranian patients with MiRAS
\end{abstract}

This study was supported by a grant from Tehran University of Medical Sciences.

Mailing Address: Nima Rezaei, MD., PhD., Children's Medical Center Hospital, Dr. Gharib St., Keshavarz Blvd., Tehran, Iran; Phone: +9821-669 292 34;

Fax: +9821-669 292 35; e-mail: rezaei_nima@tums.ac.ir 
for IL-2 gene ( $G-330 \mathrm{~T})$ and (G +166 T) and IFN- $\gamma$ gene at position UTR5644 $(A / T)$. Frequency of each allele and genotype was compared with control group. IL-2 +166 G allele was significantly lower among patients which was reflected in significantly decreased of GG genotype at this position, while IL-2 $+166 \mathrm{~T}$ allele was significantly higher among patients, IL-2 GT genotype was also significantly higher in RAS patients. No significant differences were found regarding IL-2 -330 G/T allele frequencies, while IL-2 GT genotype at this position was significantly higher among patients and IL-2 -330 TT genotype was significantly lower among RAS patients. Although no significant differences were found in IFN- $\gamma$ allele frequencies at UTR5644 (A/T), AT genotype at this position was significantly overrepresented among patients compared with controls. Results of this study suggest that certain SNPs of IL-2 and IFN- $\gamma$ genes have association with predisposition of individuals to RAS. More studies in different ethnic groups are needed to confirm results of this study.

\section{Introduction}

Recurrent aphthous stomatitis (RAS) is the most common oral painful ulcerative inflammatory disease of unknown etiology (Preeti et al., 2011). Three clinical manifestations have been described for RAS: Minor (MiRAS), Major (MaRAS), Herpetiform Ulcers (HU). MiRAS is the most common manifestation which affects about $80 \%$ of RAS patients (Bazrafshani et al., 2002a). Although the precise etiology of RAS has not been understood yet, it has been designated that some immune dysfunction may underlie RAS pathogenesis (Chavan et al., 2012). Compelling evidences demonstrate that a dysregulated, local, cell-mediated immune response, which conduce to accumulation of T-cells and leads to tissue breakdown, might be responsible for formation of RAS ulcers (Akintoye and Greenberg, 2005).

An increased systematic production of Th1 cytokines and local transcription of Th1 genes have been reported in patients with RAS (Borra et al., 2004; Lewkowicz et al., 2005). Interleukin-2 (IL-2) and Interferon-gamma (IFN- $\gamma$ ) are two cytokines secreted by Th1 cells, which are considered as pro-inflammatory cytokines the elevated levels of both have been reported in RAS patients (Albanidou-Farmaki et al., 2007; Chavan et al., 2012). Treating CD4+ cells with IFN- $\gamma$ may lead to Th2-to-Th1 exchange, and as a result of grow inhibitory of IFN- $\gamma$, the number of Th2 cells decreases while Th1 cells proliferate (Schroder et al., 2004).

IL-2 and IFN- $\gamma$ might be strong candidates for involvement with RAS pathogenesis. Cytokine gene polymorphism plays an essential role in cytokine secretion. This study was designed to evaluate the possible single nucleotide polymorphisms (SNPs) in the gene of IL-2 at positions $+166(\mathrm{G} / \mathrm{T}),-330(\mathrm{G} / \mathrm{T})$ and also in the gene of IFN- $\gamma$ at position UTR5644 (A/T) in a sample of Iranian patients with MiRAS. To our best knowledge, this is the first investigation of IL-2 and IFN- $\gamma$ SNPs in individuals with MiRAS. 


\section{Material and Methods}

Subjects

The Ethical Committee of Tehran University of Medical Sciences approved this project. Written informed consent was obtained from all subjects included to this study before sampling. Five $\mathrm{ml}$ of blood was obtained from sixty-four Iranian patients with MiRAS (24 men and 40 women) from the Department of Oral Medicine, School of Dentistry of Tehran University of Medical Sciences. 23 cases have less than three aphthous episodes per month, while 41 cases had 3 or more aphthous episodes per month. 140 age, sex and ethnicity matched healthy controls (101 men and 40 women), who were randomly selected to be enrolled in this study.

All patients were assessed by an oral medicine specialist and diagnosis of RAS was made based on accepted international clinical criteria (Ship et al., 2000). Neither patients nor controls had history of smoking or any systemic diseases such as Behcet's syndrome, diabetes mellitus, PFAPA syndrome, HIV infection. History of exposure to radiation, drugs consumption, and pregnancy at the time of study and any other periodontal diseases considered as exclusion criteria for both experimental and control groups.

\section{Genotyping}

DNA was isolated using phenol-chloroform method. IL-2 and IFN- $\gamma$ gene typing was done by polymerase chain reaction with sequence-specific primers (PCR-SSP) assay (PCR-SSP kit, Heidelberg University, Heidelberg, Germany), the method described in detail previously (Amirzargar et al., 2008). Briefly, amplification was carried out, using a thermal cycler Techne Flexigene apparatus, and the presence or absence of PCR product was visualized by $2 \%$ agarose gel electrophoresis. After electrophoresis, the gel was placed on a UV transilluminator and a picture for interpretation and documentation was taken. The allele and genotype frequencies of IL-2 (G -330 T), (G +166 T) and IFN- $\gamma($ UTR5644 A/T) were determined.

\section{Statistics}

Data analysis was performed using SPSS statistical software package (version 15.0). Chi-square test was used to compare frequencies of alleles, genotypes and haplotypes between patients and control groups. The odds ratio (OR) and $95 \%$ confidence intervals $(\mathrm{Cl})$ were calculated. Comparison of medians of quantitative variables was performed, using Mann-Whitney U-test. P-value (P) of $<0.05$ was considered significant. Allele frequencies were estimated by direct gene counting.

\section{Results}

Alleles, genotypes and haplotype frequencies

IL-2 and IFN- $\gamma$ allelic and genotype frequencies in RAS patients and healthy controls are shown in Table 1. IL-2 haplotype frequency also is presented in Table 1. 
Table 1 - Comparison of alleles, genotypes and haplotypes frequencies of IL-2 and IFN- $\gamma$ between patients with RAS and the control group

\begin{tabular}{|c|c|c|c|c|c|}
\hline $\begin{array}{l}\text { Cytokine } \\
\text { position }\end{array}$ & $\begin{array}{c}\text { Alleles/ } \\
\text { genotypes/ } \\
\text { haplotypes }\end{array}$ & $\begin{array}{c}\text { RAS } \\
(n=60), \\
n(\%)\end{array}$ & $\begin{array}{l}\text { Controls } \\
(n=140) \\
n(\%)\end{array}$ & P-value & $\begin{array}{c}\text { Odds ratio } \\
\text { (95\% confidence } \\
\text { interval) }\end{array}$ \\
\hline \multirow[t]{5}{*}{ IL-2 -330 } & G & $58(48.3)$ & $110(39.6)$ & 0.1290 & $1.43(0.91-2.25)$ \\
\hline & $\mathrm{T}$ & $62(51.7)$ & $168(60.4)$ & 0.1290 & $0.70(0.44-1.10)$ \\
\hline & GG & $3(5.0)$ & $8(5.8)$ & 0.5650 & $0.86(0.17-3.75)$ \\
\hline & GT & $52(86.7)$ & $94(67.6)$ & 0.0089 & $3.11(1.29-7.76)$ \\
\hline & TT & $5(8.3)$ & $37(26.6)$ & 0.0066 & $0.25(0.08-0.72)$ \\
\hline \multirow[t]{5}{*}{ IL-2 +166 } & G & $80(66.7)$ & $219(78.8)$ & 0.0147 & $0.54(0.33-0.89)$ \\
\hline & $\mathrm{T}$ & $40(33.3)$ & $59(21.2)$ & 0.0147 & $1.86(1.12-3.07)$ \\
\hline & GG & $22(36.7)$ & $82(59.0)$ & 0.0061 & $0.40(0.21-0.79)$ \\
\hline & GT & $36(60.0)$ & $55(39.6)$ & 0.0124 & $2.29(1.18-4.46)$ \\
\hline & $\mathrm{TT}$ & $2(3.3)$ & $2(1.4)$ & 0.3500 & $2.36(0.23-24.15)$ \\
\hline \multirow[t]{4}{*}{$-330,+166$} & GG & $54(29.8)$ & $107(38.8)$ & 0.0635 & $0.67(0.44-1.02)$ \\
\hline & TG & $55(30.4)$ & $112(40.6)$ & 0.0345 & $0.64(0.42-0.97)$ \\
\hline & TT & $37(20.5)$ & $56(20.3)$ & 0.9360 & $1.01(0.62-1.65)$ \\
\hline & GT & $35(19.3)$ & $1(0.3)$ & 0.0000 & $65.92(9.57-1307.56)$ \\
\hline IFN- $\gamma$ & $A$ & $62(51.7)$ & $140(50.7)$ & 0.9490 & $1.04(0.66-1.63)$ \\
\hline \multirow[t]{4}{*}{ UTR5644 } & $\mathrm{T}$ & $58(48.3)$ & $136(49.3)$ & 0.9490 & $0.96(0.61-1.51)$ \\
\hline & AA & $14(23.3)$ & $43(31.2)$ & 0.3430 & $0.67(0.31-1.42)$ \\
\hline & AT & $34(56.7)$ & $54(39.1)$ & 0.0330 & $2.03(1.05-3.94)$ \\
\hline & TT & $12(20.0)$ & $41(29.7)$ & 0.2130 & $0.59(0.27-1.29)$ \\
\hline
\end{tabular}

RAS - recurrent aphthous stomatitis

\section{IL-2 polymorphisms}

IL-2 +166 G allele was significantly lower $(\mathrm{OR}=0.54, \mathrm{Cl}=0.33-0.89, \mathrm{p}=0.014)$ in RAS patients compared to controls, which was reflected in significantly lower GG genotype $(\mathrm{OR}=0.40, \mathrm{Cl}=0.21-0.79, \mathrm{p}=0.061)$ at this position. IL-2 $+166 \mathrm{~T}$ allele was significantly higher among patients $(\mathrm{OR}=1.86, \mathrm{Cl}=1.21-3.07, \mathrm{p}=0.014)$. TT genotype at this position was more common among patients but did not reach significance $(p=0.3)$.

While IL-2 G +166 allele was significantly lower among patients and IL-2 T +166 allele was significantly higher among study group, IL-2 GT genotype at the same position was significantly higher $(O R=2.29, C l=1.18-4.46, p=0.012)$ among $R A S$ patients in comparison with healthy controls.

There was no significant differences in IL-2 (G -330 T) allele frequencies, while IL-2 GT genotype at position -330 was significantly higher $(O R=3.11$, $\mathrm{Cl}=1.29-7.79, \mathrm{p}=0.0089)$ among patients compared with controls. TT genotype at the same position was significantly lower $(\mathrm{OR}=0.25, \mathrm{Cl}=0.08-0.72, \mathrm{p}=0.0066)$ among RAS patients compared with controls. 
Comparison of IL-2 haplotype frequencies between the patients and controls indicated that TG haplotypes were significantly lower $(\mathrm{OR}=0.64, \mathrm{Cl}=0.42-0.97$, $p=0.03$ ) in the patient group, while GT haplotype was significantly higher $(\mathrm{OR}=65.92, \mathrm{Cl}=9.57-1307.56, \mathrm{p}=0.0000)$ among patients with RAS.

\section{IFN- $\gamma$ polymorphisms}

There was no significant differences in IFN- $\gamma$ allele frequencies at UTR5644 (A/T), while IFN- $\gamma$ AT genotype at the same position was significantly overrepresented $(\mathrm{OR}=2.03, \mathrm{Cl}=1.05-3.94, \mathrm{p}=0.033)$ among study group compared with healthy controls.

\section{Discussion}

Although association of a number of cytokines gene polymorphisms with RAS have been investigated (Najafi et al., 2014, 2015), to our best knowledge, this is the first time that association of IL-2 and IFN- $\gamma$ gene polymorphisms with RAS is reported. Buno et al. (1998) demonstrated elevated levels of IL-2 and IFN- $\gamma$ mRNA in RAS lesions, consistent with a cell-mediated immune response, thereby formation of aphthous ulcers is mainly dependent on the activation of the Th1-type immune response, which leads to elevated levels of Th1 cytokines such as IL-2 and IFN- $\gamma$ (Buno et al., 1998; Lewkowicz et al., 2011).

To the best of our knowledge, we for the first time investigated IL-2 and IFN- $\gamma$ SNPs in RAS. Some significant differences in alleles, genotype and haplotypes were found between study patients and controls. IL-2 SNPs were investigated at positions $-330(\mathrm{G} / \mathrm{T})$ and $+166(\mathrm{G} / \mathrm{T})$ in RAS patients and healthy controls. The results of our investigation indicated that $G+166$ allele was significantly lower in the patient group, while $T+166$ allele was significantly higher among patients. The +166 GG genotype and -330 TT genotype were significantly lower among the patient group. While IL-2 -330 GT genotype and +166 GT genotype were significantly higher among RAS patients, it seems that individuals who are heterogeneous in these positions are predisposed to RAS. The results of this study illustrates that the G to T exchange at position +166 of IL-2 gene might be responsible for increased IL-2 secretion.

The distribution of IFN- $\gamma$ AT genotype at position UTR5644 was significantly higher among RAS patients compared with controls, indicating that UTR5644 AT genotype is likely to be responsible for the elevated level of IFN- $\gamma$ in RAS patients (Buno et al., 1998; Lewkowicz et al., 2011). It seems that heterogeneity at this position of the IFN- $\gamma$ gene predisposed individuals to RAS.

A Th1 type immune response has been shown in RAS pathogenesis (Bazrafshani et al., 2003), which eradicates pathogens and also cause immunopathology. Elevated levels of Th1 cytokines such as tumour necrosis factor (TNF), IL-2 and IFN- $\gamma$ and on the other hand lower levels of IL-4, IL-10 and IL-5 which are secreted by Th2 cells in patients with RAS, support this hypothesis (Buno et al., 1998). Bazrafshani 
et al. (2002b) have demonstrated no significant association between RAS and TNF polymorphisms. These findings support the notion that genetic factors related to Th1 type immune response more likely operating through the IFN- $\gamma$ and IL-2 polymorphisms, and genetically predispose individuals to RAS. Indeed more studies in other ethnic groups and larger populations are needed to confirm these findings.

\section{References}

Akintoye, S. O., Greenberg, M. S. (2005) Recurrent aphthous stomatitis. Dent. Clin. North Am. 49, $31-47$.

Albanidou-Farmaki, E., Markopoulos, A. K., Kalogerakou, F., Antoniades, D. Z. (2007) Detection, enumeration and characterization of $\mathrm{T}$ helper cells secreting type 1 and type 2 cytokines in patients with recurrent aphthous stomatitis. Tohoku J. Exp. Med. 212, 101-105.

Amirzargar, A. A., Naroueynejad, M., Khosravi, F., Dianat, S. S., Rezaei, N., Mytilineos, J., Nikbin, B. (2008) Cytokine single nucleotide polymorphisms in Iranian populations. Eur. Cytokine Netw. 19, 104-112.

Bazrafshani, M. R., Hajeer, A. H., Ollier, W. E., Thornhill, M. H. (2002a) IL-1B and IL-6 gene polymorphisms encode significant risk for the development of recurrent aphthous stomatitis (RAS). Genes Immun. 3, 302-305.

Bazrafshani, M. R., Hajeer, A. H., Ollier,W. E., Thornhill, M. H. (2002b) Recurrent aphthous stomatitis and gene polymorphisms for the inflammatory markers TNF-alpha ,TNF-beta and the vitamin D receptor: no association detected. Oral Dis. 8, 303-307.

Bazrafshani, M. R., Hajeer, A. H., Ollier,W. E., Thornhill, M. H. (2003) Polymorphisms in the IL-10 and IL-12 gene cluster and risk of developing recurrent aphthous stomatitis. Oral Dis. 9, 287-291.

Borra, R. C., Andrade, P. M., Silva, I. D., Morgun, A., Weckx, L. L., Smirnova, A. S., Franco, M. (2004) The Th1/Th2 immune type response of the recurrent aphthous ulceration analyzed by cDNA microarray. J. Oral Pathol. Med. 33, 140-146.

Buno, I. J., Huff, J. C., Weston, W. L., Cook, D. T., Brice, S. L. (1998) Elevated levels of interferon gamma, tumor necrosis factor alpha, interleukins 2,4 , and 5 , but not interleukin 10 , are present in recurrent aphthous stomatitis. Arch. Dermatol. 134, 827-831.

Chavan, M., Jain, H., Diwan, N., Khedkar, S., Shete, A., Durkar, S. (2012) Recurrent aphthous stomatitis: a review. J. Oral Pathol. Med. 41, 577-583.

Lewkowicz, N., Lewkowicz, P., Banasik, M., Kurnatowska, A., Tchorzewski, H. (2005) Predominance of type 1 cytokines and decreased number of CD4(+)CD25(+high) T regulatory cells in peripheral blood of patients with recurrent aphthous ulcerations. Immunol. Lett. 99, 57-62.

Lewkowicz, N., Kur, B., Kurnatowska, A., Tchorzewski, H., Lewkowicz, P. (2011) Expression of Th1/Th2/Th3/ Th17-related genes in recurrent aphthous ulcers. Arch. Immunol. Ther. Exp. (Warsz.) 59, 399-406.

Najafi, S., Firooze Moqadam, I., Mohammadzadeh, M., Bidoki, A. Z., Yousefi, H., Farhadi, E., Tonekaboni, A., Meighani, G., Amirzargar, A. A., Rezaei, N. (2014) Interleukin-10 gene polymorphisms in recurrent aphthous stomatitis. Immunol. Invest. 43, 405-409.

Najafi, S., Yousefi, H., Mohammadzadeh, M., Bidoki, A. Z., Firouze Moqadam, I., Farhadi, E., Amirzargar, A. A., Rezaei, N. (2015) Association study of interleukin-1 family and interleukin-6 gene single nucleotide polymorphisms in recurrent aphthous stomatitis. Int. J. Immunogenet. 42, 428-431.

Preeti, L., Magesh, K., Rajkumar, K., Karthik, R. (2011) Recurrent aphthous stomatitis. J. Oral Maxillofac. Pathol. 15, 252-256.

Schroder, K., Hertzog, P. J., Ravasi, T., Hume, D. A. (2004) Interferon-gamma: an overview of signals, mechanisms and functions. J. Lekoc. Biol. 75, 163-189.

Ship, J.A., Chavez, E. M., Doerr, P. A., Henson, B. S., Sarmadi, M. (2000) Recurrent aphthous stomatitis. Quintessence Int. 31, 95-112.

Najafi S.; Yousefi H.; Mohammadzadeh M.; Zare Bidoki A.; Farhadi E.; Rezaei N. 